\title{
Expression of Receptors for SARS-CoV-2 in the Gut of Patients with Inflammatory Bowel Disease
}

\author{
Giovanni Monteleone, Eleonora Franzè, and Federica Laudisi \\ Department of Systems Medicine, University of Rome Tor Vergata, Rome, Italy
}

\section{To the Editor:}

Coronavirus disease 2019 (COVID-19) is a new infectious respiratory disease caused by severe acute respiratory syndrome coronavirus-2 (SARS-CoV-2), one of the seven coronaviruses known to infect humans. Because of the human-to-human transmission route, as of March 25, 2020, 38,0000 subjects have been diagnosed with SARS-CoV-2, and more than 10,000 infected patients have died worldwide. The clinical spectrum of SARS-CoV-2 ranges from asymptomatic or mild respiratory disease to pneumonia with respiratory distress syndrome and/or sepsis, which can result in a fatal outcome. Common symptoms are fever, cough, and shortness of breath, but gastrointestinal symptoms can occur in some patients. ${ }^{1}$ SARS-CoV-2 infection is especially frequent in the elderly population and in people with coexisting pathologies and weakened immune systems. The latter could include patients with Crohn's disease (CD) and patients with ulcerative colitis (UC), the major inflammatory bowel diseases (IBDs) in human beings, as these pathologies are associated with an increased risk of complications, including infections, due to concurrent therapy with immunosuppressive drugs and/or the chronicity of gut inflammation. ${ }^{2}$

To gain entry into cells, SARS-CoV-2 uses angiotensin-converting enzyme 2 (ACE2), a protein that is highly expressed in the human intestine. ${ }^{3}$ Evaluation of the distribution of the virus in human biological samples revealed that more than half of the fecal samples of patients with COVID-19-driven pneumonia were positive for SARS-CoV-2, and about one-fifth of infected patients remained positive in stools after becoming negative in respiratory samples. ${ }^{4}$ These observations suggest that human intestinal tract could serve as an alternative infection route for SARS-CoV-2. If this is true, we could hypothesize that patients with active IBD face an increased risk of SARS-CoV-2 infection, as inflammation can enhance ACE2 expression. We performed a gene array of biopsy samples taken from the inflamed colonic mucosa of six patients with $\mathrm{CD}$, seven patients with UC and six normal controls and initially compared the expression of ACE2 between inflamed and uninflamed samples. Four IBD patients were on mesalazine, three were on steroids and the remaining were off therapy. The study was approved by the local Ethics Committee (protocol number: 154/12). ACE2 abundance was 1.3 times higher in $\mathrm{CD}$ and 1.27 times lower in UC, respectively than in the control samples (Fig. 1). Although transmission of SARS-CoV-2 occurs primarily via respiratory droplets, the content of ACE2 is rather low in the lung compared to other human tissues, thus suggesting that SARS-CoV-2 infection also relies on coreceptors and/or other factors expressed by the host. ${ }^{5}$ Analysis of these factors in IBD tissues revealed that ANPEP, alanyl aminopeptidase, a host receptor targeted by many

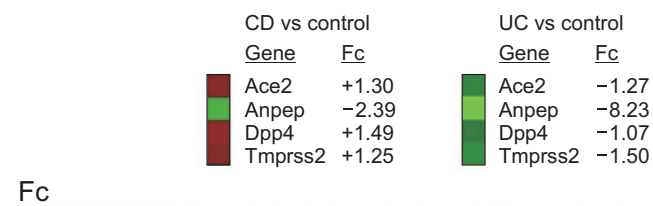

Minimum $=-9.00 \quad 0.00 \quad$ Maximum $=9.00$

Fig. 1. Heat map showing microarray based differential expression, log2 (fold change, Fc) of ACE2, ANPEP, DPP4 and TMPRSS2 genes in colonic biopsy samples isolated from inflamed tissues of patients with Crohn's disease (CD), patients with ulcerative colitis (UC) and macroscopically and microscopically unaffected colonic mucosa of patients undergoing colon cancer screening (control). Total RNA was extracted using RNeasy Mini Kit with RNase free DNase set (Qiagen, Milan, Italy). Samples with quantified complementary DNA were sequenced in Microarray Unit of Cogentech (Milan, Italy). Hybridization was performed using the GeneChip ${ }^{\circledR}$ Hybridization, Wash and Stain Kit (Affymetrix; Thermo Fisher Scientific, Monza, Italy). Transcripts were selected on base of Fc value $\geq 1.5$ or $\leq 1.5$. All the transcripts present on GeneChip ${ }^{\circledR}$ array were mapped to related classes by Gene Ontology which gave the Fc generated from comparison between $\mathrm{CD}$ versus control and UC versus control.

Correspondence to: Giovanni Monteleone

Department of Systems Medicine, University of Rome Tor Vergata, Via Montpellier, 1-00133 Rome, Italy

Tel: +39-6-72596158, Fax: +39-6-72596391, E-mail: Gi.Monteleone@Med.uniroma2.it

Received on March 26, 2020. Revised on April 9, 2020. Accepted on April 16, 2020. Published online June 3, 2020. pISSN 1976-2283 eISSN 2005-1212 https://doi.org/10.5009/gnl20112

(a) This is an Open Access article distributed under the terms of the Creative Commons Attribution Non-Commercial License (http://creativecommons.org/licenses/by-nc/4.0) which permits unrestricted non-commercial use, distribution, and reproduction in any medium, provided the original work is properly cited. 
viruses belonging to the family Coronaviridae, and DPP4, the receptor for Middle East respiratory syndrome coronavirus, were decreased in UC compared to controls. Moreover, UC samples exhibit decreased expression of the serine protease TMPRSS2, whose activity is crucial in promoting cleavage of the spike (S) protein of the virus, thereby allowing fusion of viral and cellular membranes. ${ }^{3}$ In contrast, CD samples exhibited a slight increase in the gene expression of DPP4 and TMPRSS2 and reduced expression of ANPEP compared to controls (Fig. 1). Thus, we can speculate that IBD-associated inflammation by itself does not enhance expression of the factors allowing extrarespiratory transmission of SARS-CoV-2.

\section{CONFLICTS OF INTEREST}

No potential conflict of interest relevant to this article was reported.

\section{ORCID}

Giovanni Monteleone https://orcid.org/0000-0003-1339-9076
Eleonora Franzè

https://orcid.org/0000-0003-4343-221X

Federica Laudisi

https://orcid.org/0000-0001-7380-0269

\section{REFERENCES}

1. Zhu N, Zhang D, Wang W, et al. A novel coronavirus from patients with pneumonia in China, 2019. N Engl J Med 2020;382:727-733.

2. Dulai PS, Thompson KD, Blunt HB, Dubinsky MC, Siegel CA. Risks of serious infection or lymphoma with anti-tumor necrosis factor therapy for pediatric inflammatory bowel disease: a systematic review. Clin Gastroenterol Hepatol 2014;12:1443-1451.

3. Hoffmann M, Kleine-Weber H, Schroeder S, et al. SARS-CoV-2 cell entry depends on ACE2 and TMPRSS2 and is blocked by a clinically proven protease inhibitor. Cell 2020;181:271-280.

4. Wang W, Xu Y, Gao R, et al. Detection of SARS-CoV-2 in different types of clinical specimens.JAMA 2020;323:1843-1844.

5. Qi F, Qian S, Zhang S, Zhang Z. Single cell RNA sequencing of 13 human tissues identify cell types and receptors of human coronaviruses. Biochem Biophys Res Commun 2020;526:135-140. 